\title{
On the physical origin of the quantum operator formalism
}

\author{
A. M. Cetto, L. de la Peña and A. Valdés-Hernández
}

November 25, 2020

Instituto de Física, Universidad Nacional Autónoma de México, 04510 Mexico City

\begin{abstract}
We offer a clear physical explanation for the emergence of the quantum operator formalism, by revisiting the role of the vacuum field in quantum mechanics. The vacuum or random zero-point radiation field has been shown previously - using the tools of stochastic electrodynamics - to be central in allowing a particle subject to a conservative binding force to reach a stationary state of motion. Here we focus on the stationary states, and consider the role of the vacuum as a driving force. We observe that the particle responds resonantly to certain modes of the field. A proper Hamiltonian analysis of this response allows us to unequivocally trace the origin of the basic quantum commutator, $[x, p]=i \hbar$, by establishing a one-to-one correspondence between the response coefficients of $x$ and $p$ and the respective matrix elements. The (random) driving field variables disappear thus from the description, but their Hamiltonian properties become embodied in the operator formalism. The Heisenberg equations establish the dynamical relationship between the response functions.
\end{abstract}

\section{Introduction}

"Everything is still vague and unclear to me, but it seems as if the electrons will no more move on orbits." [1]

Quantum mechanics with all its merits and successes, continues to pose deep mysteries regarding the physics underlying the description. A case in point is the operator formalism, the physical meaning of which does not seem to have been essentially clarified since Heisenberg's famous letter to Pauli dated June 1925, despite the impressive developments of the theory. Given such lack of clarity, any serious effort to throw light on this long standing matter warrants close attention. The present paper is intended as a contribution to this effort.

For this purpose we analyse the problem within the framework of nonrelativistic quantum mechanics, from the perspective provided by stochastic electrodynamics, SED (see [2]-[5] for reviews on SED). Developed on the premise 
that (otherwise classical) mechanical systems are embedded in the vacuum or zero-point radiation field ( $\mathrm{ZPF})$, taken as a real field with an energy $\frac{1}{2} \hbar \omega$ per normal mode, SED has produced a significant series of results in accordance with quantum mechanics, suggesting that the action of the field on matter is responsible for the emergence of quantization.

In this work we revisit the effect of the ZPF on the particle dynamics, with the aim to throw light on the physical origin and meaning of the quantum operator formalism. Specifically, we focus our attention on the driving effect of the field on the particle once it has reached a stationary state. In order to pave the way for the solution of the general dynamical problem, we analyse first the essence of the solution of the harmonic oscillator, the stationary part of which is characterized by its resonant response to the ZPF. In the general case of a nonlinear binding force, the system in a given stationary state $n$ responds resonantly to a set of field modes of different frequencies $\omega_{k n}$. By applying the appropriate Hamiltonian treatment, on the basis that the field has taken control of the response, we obtain the basic quantum commutator $[x, p]$ as a bilinear expression involving the corresponding response functions, which are identified with the matrix elements of the quantum operators. The Heisenberg equations of motion for the operators follow as a natural consequence.

It should be noted that, although our point of departure is the equation of motion for a charged particle in the presence of the ZPF, which is the usual starting point in SED, the paper is self-contained in the sense that to follow the arguments, the reader is not required to be familiar with the details of SED or with the process leading to the quantum results obtained so far.

\section{Statement of the problem}

We recall that the usual starting point in SED is the nonrelativistic equation of motion for a particle of mass $m$ and charge $e$ (typically an electron) subject to a conservative force $f(x)$ and to the electric component of the random zero-point radiation field in the long-wavelength approximation, $E(t)$. With the particle's radiation reaction included, the equation reads [2, 3] (we shall work throughout in one dimension, for simplicity)

$$
m \ddot{x}-f(x)-m \tau \dddot{x}=e E(t),
$$

with $p=m \dot{x}$ and $\tau=2 e^{2} / 3 m c^{3}$. The ZPF is a random stationary field with an energy $\hbar \omega / 2$ per normal mode. Since the energy of every mode is fixed, the only random element is the phase of the mode; it is because of this randomness that the problem becomes stochastic. The Fourier transform of $E(t)$ can therefore be written as

$$
\frac{1}{2 \pi} \int d t E(t) e^{i \omega t}=\widetilde{E}(\omega) a(\omega)
$$

with

$$
|\widetilde{E}(\omega)|=\frac{1}{2 \pi} \sqrt{\frac{\hbar|\omega|}{2}}, a(\omega)=e^{i \phi}
$$


and $\phi$ a random phase. Note incidentally that $a, a^{*}$ represent precisely the normal variables conventionally used for the description of a classical radiation field in reciprocal space (see, e. g., [6]). The expression $a(\omega)$ is used here to stress that to each frequency $\omega$ there corresponds a different field mode with an independent random phase, without this implying a functional dependence on $\omega$.

Let us first consider the specific case of a harmonic oscillator of natural frequency $\omega_{0}$, for which Eq. (1) can be solved exactly. As is well known the solution $x(t)$ consists of two parts: the transient one, decaying exponentially at a rate given by $\gamma=\tau \omega_{0}^{2}$, and the stationary one, driven by the field $E(t)$. Oscillators, atoms and other systems under consideration have been connected to the field for times much longer than $\gamma^{-1}$, so that the transient has disappeared and we are left with the stationary part of $x(t)$, which is

$$
x(t)=\frac{e}{m} \int d \omega \frac{\widetilde{E}(\omega)}{\omega_{0}^{2}-\omega^{2}-i \gamma \omega} a(\omega) e^{-i \omega t}+\text { c.c. }
$$

Notice from this equation that the oscillator's motion is under the control of the ZPF, to which it responds linearly. Given the fact that the ratio

$$
Q=\frac{\omega_{0}}{\gamma}=\frac{1}{\tau \omega_{0}}
$$

is very large for a typical atomic oscillator $\left(Q \sim 10^{16}\right.$ for optical frequencies), the resonant response to the field is extremely peaked. It is important to note that this resonant response is a property of the mechanical system, i. e. of the oscillator embedded in the ZPF, which will also manifest itself when it is excited by an applied field having a mode of frequency close to $\omega_{0}$; in such case the oscillator may acquire an additional energy through the excitation, thereby changing its state of motion.

\section{The nonlinear force problem}

We now extend the discussion to the general case of a particle being subject to a nonlinear conservative binding force $f(x)$ and having reached a stationary state of motion 1 The specificities of the possible stationary states of motion are determined of course by $f(x)$. What is relevant to the present discussion is that once the particle is in a stationary state, the ZPF takes over and drives the response of the system. As a typification of the adduced situation, from solidstate physics we know that materials subject to applied radiation fields - which are normally of high intensity compared with the ZPF - respond linearly to such fields, to a very good approximation 2 We may therefore safely assume that

\footnotetext{
${ }^{1}$ In SED ([2], or 3$]$ Ch. 5, it is shown that such state of motion is reached as a result of the combined effect of the background field and radiation reaction, as in the case of the harmonic oscillator shown above.

${ }^{2}$ Quoting from Ref. [7]: "Typically, the external (field) force used in experiments is small with respect to the internal ones (in a crystal), so that the system is weakly perturbed. Thus,
} 
the particle responds also linearly to the zero-point component of the radiation field.

The appropriate tools to study the response of the system to the influence of a stationary driving force are provided by the linear-response theory (LRT). An analysis of the response to different frequency components of this force is known to provide important information about the properties of the system itself [8]. Guided by the LRT, for our system in a stationary state we write the change produced by the driving force $e E(t)$ on the dynamical variable $x$ as

$$
x(t)=\frac{e}{m} \int_{-\infty}^{+\infty} d t^{\prime} \chi\left(t-t^{\prime}\right) E\left(t^{\prime}\right) .
$$

Given the stationarity of the problem it is convenient to express (6) in terms of the Fourier component of the stimulus,

$$
\widetilde{x}(\omega)=\frac{e}{m} \widetilde{\chi}(\omega) \widetilde{E}(\omega) a(\omega) .
$$

This is a central proposal, indicating that the response of the system is local in the reciprocal space. The validity of this expression is verified by direct application of the property of Maxwell's equations coupled to matter by charges and currents, whose four-dimensional Fourier transform is local in the reciprocal space $k$ [6]; here we are applying it to the fourth component of $k_{\mu}$, namely the frequency.

In the case of the harmonic oscillator, the response function $\widetilde{\chi}(\omega)$ is peaked around its natural frequency $\omega_{0}$, which is its single resonance frequency, as indicated by Eq. (44). In the general case, given the nonlinearity of the force $f(x)$ one should expect that the particle is able to respond to more than one frequency 3 whence we write the response function as $\tilde{\chi}(\omega)=\sum_{k} \tilde{\chi}_{k}(\omega)$, where

$$
\tilde{\chi}_{k}(\omega)=\frac{1}{\omega_{k}^{2}-\omega^{2}-i \gamma_{k} \omega}
$$

and the set of frequencies $\left\{\omega_{k}\right\}$ is determined by the specific form of $f(x)$, as will become clear later. The (inverse) Fourier transform of the response function is then

$$
\chi(t)=\frac{1}{2 \pi} \int_{-\infty}^{+\infty} d \omega e^{-i \omega t} \sum_{k} \tilde{\chi}_{k}(\omega),
$$

the dominant term is the linear response function. If we are able to disentangle it, the linearresponse function returns us information on the ground state and the excitation spectrum, their symmetry properties, the strength of correlations." It may of course happen that the intensity of the applied field is so high (as is the case with current high-intensity laser pulses) that the response of the system to it becomes nonlinear. This case falls beyond the scope of the present discussion.

${ }^{3}$ In fact, materials are known to resonate in general to a series of frequencies, but the responses are usually analysed separately, for simplicity. 
with $\operatorname{Re} \widetilde{\chi}_{k}(\omega)$ representing the reactive terms, and $\operatorname{Im} \widetilde{\chi}_{k}(\omega)$ the dissipative or absorptive terms.

According to the Kramers-Kronig relation [8],

$$
\widetilde{\chi}_{k}(\omega)=\int_{-\infty}^{+\infty} \frac{d \omega^{\prime}}{\pi} \frac{\operatorname{Im} \tilde{\chi}_{k}\left(\omega^{\prime}\right)}{\omega^{\prime}-\omega_{k}-i \gamma_{k} \omega},
$$

the higher the reactive response to a given frequency $\omega_{k}$, the more intense tends to be the participation of the system in absorption or emission processes at that frequency. This means that the system is able to absorb or emit energy when it is perturbed by sources that contain frequencies close to $\omega_{k}$. The new state reached by the system will of course depend on the specific absorption or emission taking place, i. e., on the frequency $\omega_{k}$. Therefore, the field mode denoted by $k$ connects the initial and final states.

Since the response functions are highly peaked, we may replace them with their value at the resonance frequency and write instead of (6)

$$
x(t)=\frac{e}{m} \sum_{k} \chi_{k} E_{k} a_{k} e^{-i \omega_{k} t}+\text { c.c. },
$$

where $\chi_{k}$ is the coefficient of the response to the field mode of frequency $\omega_{k}$, and

$$
E_{k}\left(\omega_{k}\right)=\frac{1}{2 \pi} \sqrt{\frac{\hbar\left|\omega_{k}\right|}{2}} a_{k}
$$

with $a_{k}=e^{i \phi_{k}}$.

\section{Accounting for more than one stationary state}

When the dynamics of the system admits more than one stationary state- which is generally the case for quantum systems - the set of response coefficients to different frequencies, $\left\{\chi_{k}\right\}$, will depend in principle on the state. To specify the state we add an index $n$ to $x(t)$ and to the respective response coefficients. Equation (11) becomes thus

$$
x_{n}(t)=\frac{e}{m} \sum_{k} \chi_{n k} E_{n k} a_{n k} e^{-i \omega_{k n} t}+\text { c.c. },
$$

where

$$
E_{n k}\left(\omega_{k n}\right)=\frac{1}{2 \pi} \sqrt{\frac{\hbar\left|\omega_{k n}\right|}{2}} a_{n k},
$$

and

$$
a_{n k}=e^{i \phi_{n k}} .
$$

In Eq. (13) the reverse order of the subindices in $\omega$ has been chosen in order to adjust to the normal convention of quantum mechanics. At this point it is 
convenient to introduce the response coefficients $x_{n k}$, normalized according to Eq. (14),

$$
x_{n k}=\frac{e}{2 \pi m} \sqrt{\frac{\hbar\left|\omega_{k n}\right|}{2}} \chi_{n k},
$$

so that Eq. (13) takes the form

$$
x_{n}(t)=\sum_{k} x_{n k} a_{n k} e^{-i \omega_{k n} t}+\text { c.c., }
$$

with $\left|a_{n k}\right|=1$, according to Eq. (15). Since both $x_{n}(t)$ and $E(t)$ are real quantities, the respective coefficients satisfy

$$
x_{n k}^{*}\left(\omega_{k n}\right)=x_{n k}\left(-\omega_{k n}\right), a_{n k}^{*}\left(\omega_{k n}\right)=a_{n k}\left(-\omega_{k n}\right) .
$$

To complete the picture, from Eq. (17) and

$$
p_{n}=m \dot{x}_{n}=-i m \sum_{k} \omega_{k n} x_{n k} a_{n k} e^{-i \omega_{k n} t}+\text { c.c. }
$$

we have

$$
p_{n k}=-i m \omega_{k n} x_{n k} ; p_{n k}^{*}=i m \omega_{k n} x_{n k}^{*}=p_{n k}\left(-\omega_{k n}\right) \text {. }
$$

Let us for a moment consider a system with just two stationary states. According to our observation following Eq. (10), the field mode of frequency $\omega_{k n}$ connects state $n$ with state $k$. Therefore, if the system is instead in state $k$, the mode of frequency $\omega_{n k}$ connects it with state $n$, which implies that the two indices $n, k$ enter on an equal footing in the above formulas. The response coefficient $x_{n k}\left(\right.$ or $x_{k n}$ ) determines whether a transition $n \rightarrow k($ or $k \rightarrow n$ ) can take place; if the transition $n \rightarrow k$ involves an absorption of energy $\mathcal{E}_{n k}$, the same amount of energy is emitted in the transition $k \rightarrow n$, i.e., $\mathcal{E}_{k n}=-\mathcal{E}_{n k}$.

We note incidentally that the present derivation of Eqs. (17)-(20) confirms the legitimacy of the expressions introduced in the framework of linear stochastic electrodynamics, LSED (see [9], or [4] Ch. 5). There the results followed from the demand of ergodicity, whereas here they result from locality in frequency space, Eq. (7). They tell us that for a given (binding) force $f(x)$, and in a given (stationary) state of motion $n$, the system responds resonantly and piecemeal to a given set of frequencies $\left\{\omega_{k n}\right\}$, with response coefficients $\left\{x_{n k}\right\}$. In other words, the set $\left\{x_{n k}, \omega_{k n}\right\}$ characterizes the problem and the state of the system. In order to determine their values we need to derive the corresponding dynamical equations.

To clarify a confusion expressed from time to time, we stress that the force $f(x)$, which is nonlinear in general, is not being approximated by a linear force; it is clearly the response of the system to the driving field $E(t)$ that is linear.

\section{Algebraic structure of the solution}

If we were dealing with a classical dynamical system, consisting simply of a particle subject to the force $f(x)$, the algebraic structure would be that of the 
phase-space variables $(x, p)$, which define the Poisson bracket

$$
\{x(t), p(t)\}_{x p}=1 .
$$

In the present case, by contrast, we are dealing with the responses $x_{n}(t), p_{n}(t)$ of the system in a given state $n$ to a set of relevant field modes $\{n k\}$. As indicated by Eq. (17), these responses depend directly on the field coefficients $a_{n k}$, which are related to the phase-space variables of the respective field modes, namely on $\left\{\mathrm{q}_{n k}, \mathrm{p}_{n k}\right\}$, as follows,

$$
\mathrm{q}_{n k}=\sqrt{\frac{\hbar}{2\left|\omega_{k n}\right|}}\left(a_{n k}+a_{n k}^{*}\right), \mathrm{p}_{n k}=-i \sqrt{\frac{\hbar\left|\omega_{k n}\right|}{2}}\left(a_{n k}-a_{n k}^{*}\right) .
$$

The algebraic structure of the space of the particle's response variables must reflect this dependence on the set $\{n k\}$ of canonical field variables. This means that the Poisson bracket of $x_{n}(t)$ and $p_{n}(t)$ shall not be taken with respect to $\left\{x_{i}, p_{i}\right\}$ (or $\{x, p\}$ in the one-dimensional case), but with respect to $\left\{\mathrm{q}_{n k}, \mathrm{p}_{n k}\right\}$. We therefore write, for any stationary state $n$,

$$
\left\{x_{n}(t), p_{n}(t)\right\}_{\mathrm{qp}}=\sum_{k}\left(\frac{\partial x_{n}}{\partial \mathrm{q}_{n k}} \frac{\partial p_{n}}{\partial \mathrm{p}_{n k}}-\frac{\partial p_{n}}{\partial \mathrm{q}_{n k}} \frac{\partial x_{n}}{\partial \mathrm{p}_{n k}}\right)=1,
$$

where the subindex qp denotes the relevant set of canonical field variables. The transformation rules (22) applied to this Poisson bracket allow to express it in terms of the normal field coefficients,

$$
\left\{x_{n}(t), p_{n}(t)\right\}_{\mathrm{qp}}=-\frac{i}{\hbar} \sum_{k}\left(\frac{\partial x_{n}}{\partial a_{n k}} \frac{\partial p_{n}}{\partial a_{n k}^{*}}-\frac{\partial p_{n}}{\partial a_{n k}} \frac{\partial x_{n}}{\partial a_{n k}^{*}}\right)=1 .
$$

Therefore, this new bilinear form, which we denote with a square bracket

$$
\sum_{k}\left(\frac{\partial x_{n}}{\partial a_{n k}} \frac{\partial p_{n}}{\partial a_{n k}^{*}}-\frac{\partial p_{n}}{\partial a_{n k}} \frac{\partial x_{n}}{\partial a_{n k}^{*}}\right) \equiv\left[x_{n}, p_{n}\right]
$$

must, according to Eq. (24), satisfy the equation

$$
\left[x_{n}, p_{n}\right]=i \hbar,
$$

with $x_{n}, p_{n}$ given by (17) and (19). Since the value of this Poisson bracket does not depend on the state $n$, we may write in general

$$
[x, p]=i \hbar
$$

for $x, p$ in any state.

This result is crucial: it indicates that the scale of the response of the system to the field variables, expressed through the bilinear form (25), is universally

\footnotetext{
${ }^{4}$ This bilinear form was introduced already in [10], under the name Poissonian; see also [3] Ch. 10).
} 
determined by Planck's constant, that is, by the scale of the energy of the ZPF

modes. Notice that the energy per normal mode $\frac{1}{2} \hbar \omega$, expressed through the factor $\sqrt{\left|\omega_{n k}\right| / 2 \hbar}$ intervening in the transformation from canonical to normal variables - or, equivalently, the spectral composition of the ZPF proportional to $\omega^{3}$, which ensures its Lorentz invariance [11, 12] - plays a decisive role. Any other spectral form of the field would have led to an explicit dependence on $\omega$ in (25), which then would not have a universal value.

To close this section we note that by using Eqs. (17)-(20), the binary operation on the variables $(x, p)$ denoted by the bracket $[x, p]$ becomes expressed in terms of the response coefficients $x_{n k}$ and the respective frequencies $\omega_{n k}$,

$$
\sum_{k}\left(x_{n k} p_{n k}^{*}-p_{n k} x_{n k}^{*}\right)=2 i m \sum_{k} \omega_{k n}\left|x_{n k}\right|^{2}=i \hbar,
$$

whence

$$
2 m \sum_{k} \omega_{k n}\left|x_{n k}\right|^{2}=\hbar
$$

for any state $n$.

The process leading to (27) reveals thus one of the most intricate quantum enigmas, by exhibiting the Poisson bracket as the result of the transition from the classical+ZPF dynamics to the quantum dynamics, meaning the (Hamiltonian) dynamics describing the response of the system to the driving field modes. Let us now build on this result.

\section{Matrix mechanics; operators and the Hilbert space}

As observed in the paragraph following Eq. (20), the system is able to absorb or emit energy when it is perturbed by (field) sources that contain the resonance frequencies $\omega_{n k}$. This means that the response coefficients $x_{n k}$ connect a stationary state $n$ with a stationary state $k$, of a higher or a lower energy depending on the sign of $\operatorname{Im} \widetilde{\chi}_{n k}(\omega)$, given by Eq. (8). Therefore, the sign of $\omega_{n k}$ reverses with the interchange of $n$ and $k$, i.e.,

$$
\omega_{n k}=-\omega_{k n} .
$$

Further, from this equation and Eqs. (18) we get

$$
x_{n k}^{*}\left(\omega_{n k}\right)=x_{k n}\left(\omega_{k n}\right), p_{n k}^{*}\left(\omega_{n k}\right)=p_{k n}\left(\omega_{k n}\right), a_{n k}^{*}\left(\omega_{n k}\right)=a_{k n}\left(\omega_{k n}\right) .
$$

Therefore Eq. (28) can be written, as was promptly discovered by Born,

$$
\sum_{k}\left(x_{n k} p_{k n}-p_{n k} x_{k n}\right)=i \hbar
$$


and its expression in the form of Eq. (29) coincides then with the ThomasReiche-Kuhn sum rule.

The coefficients $x_{n k}\left(p_{n k}\right)$ have thus become the elements of a matrix $\mathbb{X}(\mathbb{P})$ with as many rows and columns as there are different states, and Eq. (32) can be written in terms of the matrix products,

$$
(\mathbb{X P}-\mathbb{P X})_{n n}=i \hbar \mathbb{I}_{n n},
$$

for any state $n$. Since the normal field variables connecting different states $n, n^{\prime}$ are independent, we have $\left(\partial a_{n k} / \partial a_{n^{\prime} k}\right)=\delta_{n n^{\prime}}$; this allows us to write Eq. (33) in the more general form

$$
(\mathbb{X P}-\mathbb{P} \mathbb{X})_{n n^{\prime}}=i \hbar \delta_{n n^{\prime}},
$$

which is just the matrix formula discovered by Heisenberg for the quantum rule, in fact the basic quantum commutator.

Therefore, the basic quantum commutator is the matrix expression of the Poisson bracket of the system's response variables with respect to the normal field variables. The matrix element $x_{n k}$ represents the (resonant) response amplitude of the system in state $n$ to the field mode $(n k)$.

Since the matrix elements connect different stationary states $n$ and $k$, the corresponding vectors are precisely the state vectors, and the dimension of the Hilbert space is determined by the total number of possible (stationary) states. Diagonal matrices represent conserved quantities which do not connect different states; the state vectors are therefore orthogonal. For the one-dimensional conservative problem studied here, the only conserved quantity is the energy, represented by the Hamiltonian operator

$$
\mathbb{H}=\frac{\mathbb{P}^{2}}{2 m}+\mathbb{V}(\mathbb{X}) .
$$

With the help of the Leibniz rule and using Eqs. (34) and (35), it is a simple exercise to derive the equations of evolution for the matrices $\mathbb{X}, \mathbb{P}$. For the first one we obtain, using Eq. (19),

$$
\frac{1}{i \hbar}[\mathbb{X}, \mathbb{H}]=\frac{\mathbb{P}}{m}=\dot{\mathbb{X}} .
$$

For the second one we get

$$
\frac{1}{i \hbar}[\mathbb{P}, \mathbb{H}]=\mathbb{F}=\dot{\mathbb{P}} .
$$

In writing the second equality we have taken into account that in the stationary states, the radiation reaction appearing in Eq. (1) is balanced by the ZPF, as observed in connection with the harmonic oscillator, which allows us to write $f_{n}(t)=m \ddot{x}_{n}(t)=\dot{p}_{n}(t)$.

To close the loop we use the fact that in its own representation $\mathbb{H}$ is diagonal, as stated previously, and write $H_{n k}=\mathscr{E}_{n} \delta_{n k}$; whence from Eqs. (19) and (36) we obtain the well-known Bohr formula for the energy involved in the transition between states $k$ and $n$,

$$
\left(\mathscr{E}_{k}-\mathscr{E}_{n}\right)=\hbar \omega_{k n} .
$$




\section{Corollary}

The matrix formulation of quantum mechanics constitutes a most compact and elegant means of describing the (resonant) response properties of a particle subject to a (binding) conservative force $f=-V^{\prime}(x)$, embedded in the ZPF and under its control. The random vacuum field has introduced a fundamental change in the description of the dynamical behavior of the system [13], reflected in the transition from the classical to the quantum Poisson bracket. Regardless of the form of the potential $V(x)$, the particle's response to the field, expressed through $\left(x_{n}(t), p_{n}(t)\right)$, is linear and becomes represented by a set of harmonic oscillators, the amplitudes and frequencies of which depend on the external force and on the state of the system. The resonance frequencies are precisely the spectral frequencies, and the response amplitudes are the corresponding matrix elements. It is clear that the pair $\left(x_{n}(t), p_{n}(t)\right)$ does not refer to the trajectory of the particle, whence there is no (true) phase-space distribution associated with it.

The migration of the description from the physical space to the abstract Hilbert space offers a physical explanation for the latter, as Lorentz, Schrödinger, and other pioneers of the quantum theory were eagerly reclaiming at the time [14. Heisenberg was of course right in stating that his matrix mechanics does not describe the orbital motion of electrons; but he did not have at hand the vacuum field discovered by Planck fifteen years earlier, to develop his theory on more physical grounds.

In a more elaborate version we shall discuss the physical implications of the results presented here.

\section{References}

[1] Heisenberg, W.: Der Teil und das Ganze, Piper, Munich (1969). Quotation reproduced in https://www.vub.be/CLEA/IQSA/history.html.

[2] Claverie, P., Diner, S.: The concept of molecular structure in quantum theory. Israel J. Chem. 19, 54 (1980).

[3] de la Peña, L., Cetto, A.M.: The Quantum Dice. Kluwer Publ., Dordrecht (1996).

[4] de la Peña, L., Cetto, A.M., Valdés-Hernández, A.: The Emerging Quantum. Springer Verlag, Berlin (2015).

[5] Boyer, T.H.: Stochastic Electrodynamics: The Closest Classical Approximation to Quantum Theory. Atoms 7:1, 29 (2019).

[6] Cohen-Tannoudji, C., Dupont-Roc, J., Grynberg, G.: Photons and Atoms. Introduction to Quantum Electrodynamics, John Wiley, NY (1989).

[7] Pavarini, E.: Linear Response Functions. In: E. Pavarini, E. Koch, D. Vollhardt, and A. Lichtenstein, eds., DMFT at 25: Infinite Dimensions 
Modeling and Simulation Vol. 4. Forschungszentrum Jülich. ISBN 978-389336-953-9 (2014), http://www.cond-mat.de/events/correl14.

[8] Tong, D.: Lectures on Kinetic Theory, Ch. 4, University of Cambridge Graduate Course (2012). https://www.damtp.cam.ac.uk/user/tong/kinetic.html.

[9] de la Peña, L., Valdés-Hernández, A., Cetto, A.M.: Quantum Mechanics as an Emergent Property of Ergodic Systems Embedded in the Zero-point Radiation Field. Found. Phys. 39, 1240-1272 (2009).

[10] de la Peña, L., Cetto, A.M.: The physics of stochastic electrodynamics. Nuovo Cim. 92B, 189 (1986).

[11] Einstein, E., L. Hopf: Statistische Untersuchung der Bewegung eines Resonators in einem Strahlungsfeld, Ann. d. Physik 338, 1105 (1910).

[12] Marshall, T.W.: Random Electrodynamics, Proc. Roy. Soc. A276, 475 (1963).

[13] Cetto, A.M., de la Peña, L., Valdés-Hernández, A.: Relevance of stochasticity for the emergence of quantization (to be published); arXiv: 2010.06780v1 [quant-ph] (2020).

[14] Bacciagaluppi, G., Valentini, A. Quantum Theory at the Crossroads. Reconsidering the 1927 Solvay Conference. Cambridge UP, Cambridge (2009). 